\title{
Treatment satisfaction in women receiving palbociclib combination therapies for advanced/metastatic breast cancer
}

\author{
Christina Darden*, ${ }^{*}$ Debanjali Mitra², David McSorley ${ }^{1}$, Kimberly Davis $^{1}$, Juliet Band ${ }^{1}$ \& \\ Shrividya lyer ${ }^{2}$ \\ ${ }^{1}$ RTI Health Solutions, Research Triangle Park, NC 27709, USA \\ 2 Pfizer, Inc., New York, NY 10017, USA \\ *Author for correspondence: Tel.: +1 919597 5101; Fax: +1 919541 7222; cdarden@rti.org
}

\begin{abstract}
Aim: To understand treatment satisfaction in patients with advanced or metastatic breast cancer receiving palbociclib plus an aromatase inhibitor or palbociclib plus fulvestrant in a real-world setting. Patients \& methods: We performed an observational, cross-sectional, web-based survey of 604 patients with self-reported hormone receptor-positive (HR+)/HER2-negative (HER2-) ABC/mBC in six countries. Results: Overall, more than $96 \%$ of patients reported the benefits of their palbociclib combination therapy met or exceeded their expectations. Patient expectations and satisfaction with therapy did not differ between patients on palbociclib plus letrozole and palbociclib plus fulvestrant, or between patients with visceral and nonvisceral metastases. Conclusion: The patients on palbociclib combination therapy reported high satisfaction scores across multiple countries.
\end{abstract}

First draft submitted: 18 July 2018; Accepted for publication: 28 August 2018; Published online: 12 September 2018

Keywords: advanced breast cancer $\bullet$ aromatase inhibitors $\bullet$ CDK4/6 $\bullet$ estrogen receptor antagonist $\bullet$ fulvestrant $\bullet$ hormone receptor-positive $\bullet$ letrozole $\bullet$ metastatic breast cancer $\bullet$ palbociclib $\bullet$ patient satisfaction

Breast cancer represents $25 \%$ of all cancers diagnosed in women globally [1,2], with about $10 \%$ of all incident cases diagnosed at a locally advanced or metastatic breast cancer (ABC/mBC) [3,4]. An estimated 50,344 new cases of metastatic breast cancer were diagnosed among women in 2013 in the USA [5]. In 2017, it is estimated that 40,610 women died of breast cancer in the USA alone [6].

Approximately 72\% of newly diagnosed breast cancer cases of known hormone receptor (HR) and HER2 subtype were HR-positive (HR+) and HER2-negative (HER2-), according to a study that collected data from population-based cancer registries that submit data to the SEER program [7]. Prior to early 2015, there had been no new first-line therapies for more than 10 years for these patients in the USA. Based on the results of two clinical trials, PALOMA 1 [8] and PALOMA 2 [9], the CDK4/6 inhibitor palbociclib in combination with an aromatase inhibitor was approved in 2015 by the US FDA for HR+/HER2- ABC/mBC patients. Palbociclib was subsequently approved in combination with the estrogen receptor antagonist fulvestrant for women who had disease progression following endocrine therapy [10] and is now available in more than 80 countries globally. Currently, two additional CDK4/6 inhibitors are also approved in the USA for this patient population [11-14].

While patients' experiences with breast cancer treatments have been studied and reported for conventional treatments [15-21], patient satisfaction with newer targeted treatments has not been studied. We used a validated measure of patient satisfaction, the Cancer Therapy Satisfaction Questionnaire (CTSQ) [22-24], to evaluate patient expectations of therapy, feelings about side effects and satisfaction with palbociclib combination therapy in six countries, post approval, in real-world settings.

\section{Methods}

Study population \& data collection

An observational, cross-sectional, web-based survey of 604 patients with self-reported HR+/HER2- ABC/mBC was performed. The study population was identified and recruited by Global Perspectives (Norwich, UK) through

Future $\because$ Medicine 
Table 1. Cancer Therapy Satisfaction Questionnaire.

\begin{tabular}{|l|l|}
\hline Domain & Description \\
\hline & Return back to normal life \\
\hline & Get rid of the cancer \\
\hline & Prevent cancer from coming back \\
\hline Feelings about side effects & Stop the cancer from spreading \\
\hline & Help you live longer \\
\hline & Palbociclib limited daily activities \\
\hline Satisfaction with therapy & Upset about the side effects \\
\hline & Taking palbociclib as difficult as expected \\
\hline & Were side effects as expected \\
\hline & Worth taking even with the side effects \\
\hline & Thinking about stopping palbociclib \\
\hline How worthwhile was palbociclib \\
\hline Adapted with permission from Pfizer (2006) [24]. & Benefits of palbociclib meet expectations \\
\hline & Satisfaction with form of palbociclib \\
\hline & Satisfaction with recent palbociclib \\
\hline & Would you take palbociclib again \\
\hline
\end{tabular}

an online panel and proprietary databases from September 2017 through January 2018. Panel members who indicated they had breast cancer (all stages) were invited to complete the screener to confirm eligibility. After their eligibility was confirmed via the web-based screener, participants provided electronic consent prior to gaining access to the questionnaire. Participants were eligible for inclusion if they were age 18 years or older and were taking palbociclib plus an $\mathrm{AI}$ or palbociclib plus fulvestrant as per the approved indication (HR+/HER2- $\mathrm{ABC} / \mathrm{mBC}$ ) for 2 or more months at the time of the survey. In Canada, eligible patients were taking palbociclib plus letrozole as initial endocrine-based therapy or palbociclib plus fulvestrant following endocrine therapy. The questionnaire was administered in six countries (USA, Canada, Germany, Netherlands, Argentina and Denmark) and was translated into the appropriate native languages for each country. RTI International's institutional review board (IRB) reviewed the study design and questionnaire and provided an exemption for the study. The study protocol conformed to the ethical guidelines of the 1975 Declaration of Helsinki. For Canada, central IRB approval was obtained. For Germany, a study notification to the Federal Institute for Drugs and Medical Devices (Bundesinstitut für Arzneimittel und Medizinprodukte, BfArM) was submitted, and approval from Freibeurger Ethik-Kommission International (FEKI) was obtained for Germany, Denmark and the Netherlands. Separate ethics submissions were not required in Argentina, Denmark or the Netherlands.

\section{Survey}

Standard survey methodological principles were used to draft items [25]. The questions focused on demographic information (age, race, educational level, employment status), medical history of breast cancer (e.g., time since first diagnosis of breast cancer, time since diagnosis of $\mathrm{ABC} / \mathrm{mBC}$, metastases) and current and past treatments. The survey also included 16 questions from the CTSQ, a validated instrument that measures satisfaction with therapy from the patient perspective. The CTSQ was developed for use in adult patients with any type of cancer, regardless of the stage and treatments used [22].

The CTSQ [22] includes questions for three domains:

- Expectations of therapy in preventing recurrence or progression, comprising five items;

- Feelings about side effects, comprising four items;

- Satisfaction with therapy, comprising seven items.

Each domain is scored from 0 to 100 , with higher scores associated with the best outcome on each domain. For this study, the patients were presented with questions in which the term 'IV/pills' was substituted with 'Ibrance (palbociclib).' Table 1 presents a description of the CTSQ domains and items. 


\section{Statistical analysis}

Responses and scores were analyzed descriptively through the tabular display of summary statistics (mean, standard deviation [SD], median, and range) for continuous variables and frequency counts and percentages for categorical variables. No imputation of missing data was performed. Domain scoring for the CTSQ was conducted per the CTSQ scoring guide [24]; 95\% confidence intervals for the mean were calculated for each CTSQ domain score.

Subgroup analyses were performed by the combination therapy used, country, age categories, prior exposure to chemotherapy and length of time since starting palbociclib and sites of metastases. Sites of metastases were categorized as visceral or nonvisceral, with visceral status defined as metastases of the brain, liver, lungs/pleura and pancreas. A participant could select more than one site of metastasis. Patients who reported metastasis to the bone, lymph nodes and skin/soft tissue were categorized as having nonvisceral metastases. Pairwise comparisons of mean CTSQ domain scores within subgroups were conducted using t-tests, and p-values were reported without adjustment for multiple comparisons.

\section{Results}

\section{Respondent characteristics}

The questionnaire was administered in six countries (USA, Canada, Germany, Netherlands, Argentina and Denmark) by RTI-HS and Global Perspectives (Norwich, UK). Overall, 2255 individuals diagnosed with breast cancer (any stage) were screened, and 642 were eligible and began the survey. Among those eligible, 604 completed the survey. Demographic and clinical data are shown in Table 2. Most of the respondents were identified as white/Caucasian (82.0\%), and half were employed (50.8\%). The median age of respondents was 41 years. Overall, mean $(\mathrm{SD})$ reported time since diagnosis of $\mathrm{ABC} / \mathrm{mBC}$ was 17.8 (42.95) months and ranged from 14.5 (8.88) months (Germany) to 23.5 (57.47) months (Argentina). Almost half of the patients $(\mathrm{n}=268 ; 44.4 \%)$ were first diagnosed with ABC (stage IIIB and IIIC), while a fourth $(\mathrm{n}=149 ; 24.7 \%)$ were first diagnosed with $\mathrm{mBC}$. The remainder of the sample comprised patients first diagnosed at an earlier stage, but who had progressed to later stage disease. The majority of patients $(83 \%)$ had previously been treated with drug(s) approved for the treatment of $\mathrm{ABC} / \mathrm{mBC}$.

Most patients $(58.6 \%)$ were taking palbociclib plus fulvestrant combination therapy at the time of completing the survey, with the remainder taking palbociclib in combination with AIs. Half of the patients (49.3\%) had been taking palbociclib for 7-12 months. Only 18.2\% had been taking palbociclib for 6 or fewer months. Descriptive statistics are reported in Table 2 for the overall cohort, by combination therapy used, and by sites of metastases (visceral or nonvisceral sites).

\section{Patient satisfaction with treatment}

Table 3 presents the CTSQ domain scores and ranges for the overall sample. On average, patients had high expectations about their therapy and reported a mean (SD) domain score of 71.80 (14.48). Most patients across all six countries $(76.8 \%)$ reported that they 'always' or 'most of the time' felt their treatment would help them 'return back to a normal life' (CTSQ item 1, data not shown). Mean domain scores for expectations of therapy were consistent across treatment groups (Figure 1). However, fewer patients with visceral metastases reported they 'always' or 'most of the time' thought their treatment would 'stop the cancer from spreading' (Figure 2, item 4) compared with patients who had no visceral metastases.

The mean (SD) score for the feelings about side effects domain was 44.73 (13.00) (Table 3). Nearly all patients $(92.2 \%)$ reported that the side effects of palbociclib were 'exactly as', 'much better', or 'somewhat better' than expected (Figure 2, item 13). The mean (SD) score for the feelings about side effects domain was higher for patients taking palbociclib plus an AI (46.88 [13.86]) compared with those taking palbociclib plus fulvestrant (43.22 [12.16], $\mathrm{p}=0.0006$ ) (Figure 1). Patients with and without visceral metastases reported similar mean scores for the feelings about the side effects domain (Figure 1). In each treatment group, and in the visceral and nonvisceral metastases subgroups, $86 \%$ or more patients indicated that side effects were 'exactly as I expected', 'somewhat better than I expected' or 'much better than I expected' (Figure 2, item 13). Patients were asked to report if taking palbociclib was as difficult as expected, and almost half (47\%) reported that taking palbociclib was 'as difficult', 'somewhat easier', or 'much easier' than they thought it would be (item 11, data not shown). Only $9.4 \%$ of patients felt that taking palbociclib was 'much more difficult' than expected.

The mean score for the satisfaction with therapy domain was higher than 66 in each of the six countries, with an overall mean score of 70.87 (10.55) (Table 3). Almost all patients (96.4\%) reported that the benefits of palbociclib 


\section{Table 2. Patient characteristics and treatment and medical history $(n=604)$.}

Variable

Age (years):

- Mean (SD)

- Median

- Min-max

Country, n (\%):

- USA

- Canada

- Germany

- Netherlands

- Argentina

- Denmark

Race or ethnicity, n (\%): ${ }^{\dagger}$

- White/Caucasian

- African/black

- Other ${ }^{\ddagger}$

- Prefer not to answer

Employment status, $\mathrm{n}(\%)$ :

- Employed full or part time

- Homemaker

- Short- or long-term inability to work due to cancer

- Other ${ }^{\S}$

- Prefer not to answer

Education level, n (\%):

- Primary education, lower secondary education or high school diploma (secondary education)

- Some college or university

- University, undergraduate degree, professional, advanced or graduate degree

- Prefer not to answer

Current treatment, $\mathrm{n}(\%)$ :

- Palbociclib + aromatase inhibitor

- Palbociclib + fulvestrant

Time since diagnosis of advanced or metastatic breast cancer (months):

$-n$

- Mean (SD)

- Median

- Min-max

Stage when breast cancer was first diagnosed, $\mathrm{n}(\%)$ :

- Early stage breast cancer (stage I, IIA, IIB and IIIA)

- Advanced breast cancer (stage IIIB and IIIC)

- Metastatic breast cancer (stage IV)

Visceral status, $\mathrm{n}(\%)$ :

$-\mathrm{n}$

- Visceral metastases

- No visceral metastases value

$41.1(7.97)$
41
$18-70$

$250(41.4)$

100 (16.6)

100 (16.6)

52 (8.6)

51 (8.4)

51 (8.4)

495 (82.0)

83 (13.7)

34 (5.6)

$2(0.3)$

\section{7 (50.8)}

200 (33.1)

35 (5.8)

69 (11.4)

$1(0.2)$

62 (10.3)

116 (19.2)

425 (70.4)

$1(0.2)$

250 (41.4)

354 (58.6)

602

17.8 (42.95)

12.1

$<1-636$

187 (31.0)

268 (44.4)

149 (24.7)

152

78 (51.3)

74 (48.7)

$\dagger$ Categories for race/ethnicity and employment status are not mutually exclusive, and more than one category can be selected.

¥Asian or Pacific Islander, Middle Eastern, Indian subcontinent (Indian, Pakistani and Bangladeshi) and other.

§Student, unemployed, retired, disabled and other.

SD: Standard deviation 

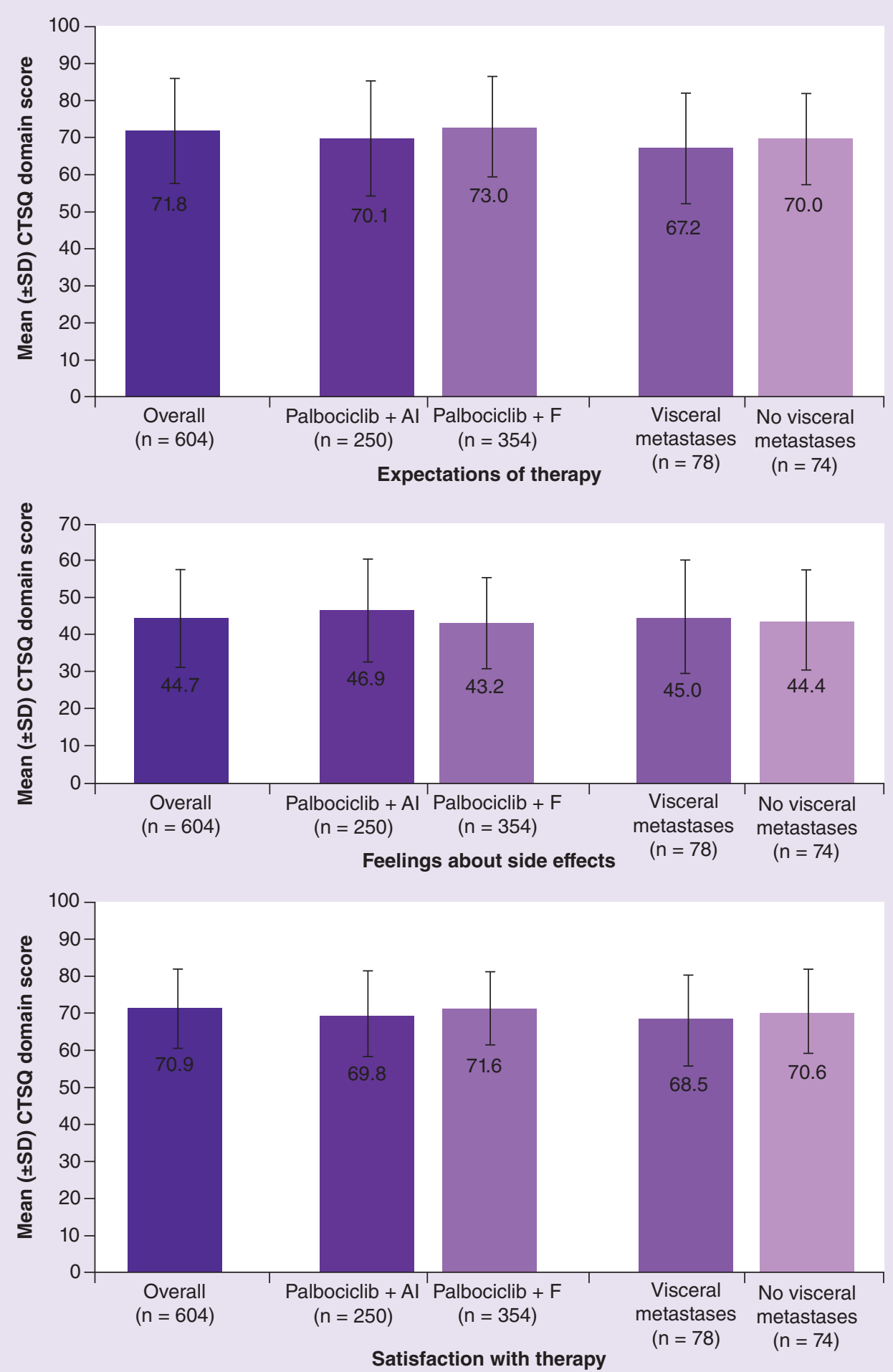

Figure 1. Cancer Therapy Satisfaction Questionnaire domain score overall, by treatment type, and by patients with and without visceral metastases.

Al: Aromatase inhibitors; CTSQ: Cancer Therapy Satisfaction Questionnaire; F: Fulvestrant; SD: Standard deviation. 
Table 3. Cancer Therapy Satisfaction Questionnaire domain scores $(n=604)$.

\begin{tabular}{|c|c|}
\hline Domain & Value \\
\hline \multicolumn{2}{|c|}{ Expectations of therapy } \\
\hline Mean (SD) & $71.80(14.48)$ \\
\hline Median & 75 \\
\hline Min-max & $25-100$ \\
\hline \multicolumn{2}{|l|}{ Range: } \\
\hline$-<25$ & $0(0)$ \\
\hline$-76-100$ & $221(36.6)$ \\
\hline \multicolumn{2}{|c|}{ Feelings about the side effects } \\
\hline Mean (SD) & $44.73(13.00)$ \\
\hline Median & 43.8 \\
\hline$-51-75$ & $136(22.5)$ \\
\hline$-76-100$ & $8(1.3)$ \\
\hline \multicolumn{2}{|c|}{ Satisfaction with therapy } \\
\hline Mean (SD) & $70.87(10.55)$ \\
\hline Median & 71.4 \\
\hline Min-max & $36-100$ \\
\hline \multicolumn{2}{|l|}{ Range: } \\
\hline$-<25$ & $0(0)$ \\
\hline$-25-50$ & $23(3.8)$ \\
\hline$-51-75$ & $420(69.5)$ \\
\hline
\end{tabular}

had 'met my expectations', had been 'somewhat better than my expectations', or had been 'much better than my expectations' (Figure 2, item 12). Almost all patients taking palbociclib in combination with AIs (90.8\%) and patients taking palbociclib in combination with fulvestrant (95.5\%) reported that they would 'probably yes' or 'yes, definitely' take palbociclib again (item 16, data not shown). Subgroup analyses were also conducted by age, country, prior exposure to chemotherapy and length of time since starting palbociclib (stratified by 2-12 months vs $>12$ months on palbociclib combination therapy), but no notable differences were found, with the exception that mean (SD) scores for the feelings about side effects domain were higher in women 50 years or older (47.94 [12.25]) compared with women 18-49 years old (44.25 [13.06], $\mathrm{p}=0.0185)$.

\section{Discussion}

Treatment options for locally $\mathrm{ABC} / \mathrm{mBC}$ are undergoing rapid change, with the introduction of new classes of therapy that target specific pathways dysregulated in breast cancer cells. In particular, palbociclib has been shown in several clinical trials to improve progression-free survival among patients with $\mathrm{ABC} / \mathrm{mBC}$ in combination with hormone therapy $[8,10]$. Current treatment guidelines in the USA, Canada and Europe recommend that postmenopausal patients diagnosed with $\mathrm{HR}+/ \mathrm{HER} 2-\mathrm{ABC} / \mathrm{mBC}$ receive first-line therapy that includes $\mathrm{CDK} 4 / 6$ inhibitors in combination with AI.

Because these treatments have been widely available for less than 3 years, little is known about patient experiences, including their feelings about side effects of treatment and their overall treatment satisfaction. Our multinational study found that, uniformly, patients reported having high expectations of therapy and satisfaction with their current treatment choice. There were no substantive differences in reported expectations of therapy, feelings about side effects or overall satisfaction among patients in any of the six countries based on the CTSQ scores. Regardless 


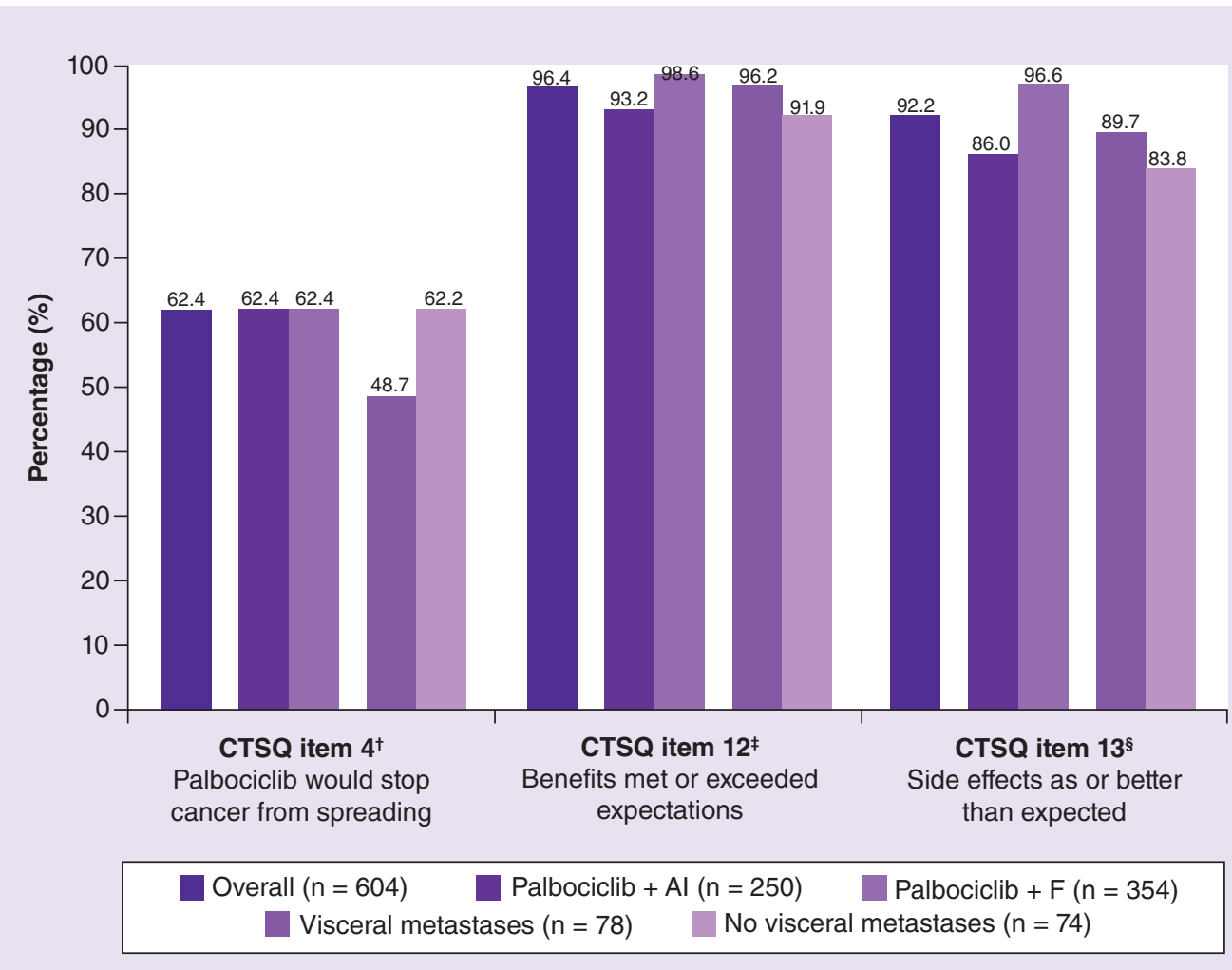

Figure 2. Cancer Therapy Satisfaction Questionnaire individual item responses.

†CTSQ item 4: "In general, in the last 4 weeks, how often did you feel that Ibrance (palbociclib) would stop the cancer from spreading?" Figure displays percentages of respondents who answered 'Always' and 'Most of the time'.

¥CTSQ item 12: "Overall, how well did the benefits of Ibrance (palbociclib) meet your expectations?" Figure displays percentages of respondents who answered 'Met my expectations', 'Somewhat better than my expectations', and 'Much better than my expectations'. $\S$ CTSQ item 13: "Overall, were the side effects of Ibrance (palbociclib) as you expected?" Figure displays percentages of respondents who answered 'Exactly as I expected', 'Somewhat better than I expected', and 'Much better than I expected'.

Al: Aromatase inhibitor; CTSQ: Cancer Therapy Satisfaction Questionnaire; F: Fulvestrant; N: Number of respondents.

of which combination therapy they were taking, patients reported that side effects were as expected or better than expected. Further, satisfaction levels were similar for combination therapy treated patients with or without visceral metastases.

In a previous study that assessed patient satisfaction with metastatic breast cancer treatments prior to the introduction of targeted treatments, the mean (SD) scores for expectations of therapy, feelings about side effects and satisfaction with therapy were $60.6(21.0), 54.9$ (17.5) and 69.4 (15.4), respectively [21]. Most patients had received either chemotherapy without endocrine therapy (46.2\%) or endocrine therapy without chemotherapy (48.8\%); 5.1\% received both or neither. The mean (SD) age of 66.3 years (10.3) was older than that of patients in the current study. Patient satisfaction scores were slightly lower than those in the current study.

To our knowledge, this is one of the first studies to describe HR+ HER 2- ABC $/ \mathrm{mBC}$ patient satisfaction with palbociclib combination treatments in a real-world setting. It revealed that, on the whole, patients with $\mathrm{ABC} / \mathrm{mBC}$ in the six countries perceive that their current palbociclib combination therapy would help stop their cancer from spreading and would help them return to a normal life. More than $96 \%$ of patients reported that the benefits of their palbociclib combination therapy met or exceeded their expectations.

In the context of patient-centered oncology care, our study provides valuable information that could help inform physician-patient conversations about the risks, benefits and potential side effects of the treatment. While shared decision making in choosing treatment options in the oncology setting has been evaluated [26,27], such studies have tended to focus on initial treatment planning. In recent years, patients have expressed interest in taking a more active role in decision making about their care [28]. Yet little information about patient experiences and 
subsequent satisfaction is available to inform those discussions. This study provides direct insight into the patient experience and can facilitate conversations between patients and physicians about their treatment. Previous research on physician and patient satisfaction with advanced breast cancer patient treatment revealed significant gaps and unmet needs [29]. The data from this study could help fill that gap for both physicians and patients, providing a reference point for treatment expectations and experiences with the risks, benefits and side effects of biologic treatments for $\mathrm{ABC} / \mathrm{mBC}$.

\title{
Limitations
}

The patient population comprised a convenience sample of patients who previously opted into a research panel. Patient respondents had a self-reported physician diagnosis of breast cancer, and no corroboration of diagnosis with medical records was performed. The incidence rate of breast cancer is almost double in women 60-64 years old globally compared with women $40-44$ years old [1]. The population who agreed to participate in this study were younger (median age: 41 years old) and more highly educated than patients diagnosed with breast cancer globally. The nature of online surveys limits the population to those with access to and the ability to use the Internet. Patients with advanced disease may not be able to complete or interested in online surveys. Therefore, our sample may not be fully representative of the entire $\mathrm{ABC} / \mathrm{mBC}$ population. Recently introduced novel therapies may influence patient opinions and offer hope of recovery or relief for those with severe illnesses. Research is ongoing and may warrant further studies that focus on patient satisfaction across a variety of therapies and timepoints.

\section{Conclusion}

Patients with $\mathrm{ABC} / \mathrm{mBC}$ currently taking combination therapy that includes palbociclib plus an $\mathrm{AI}$ or palbociclib plus fulvestrant are overall satisfied with their treatment experience. Patients reported that they would probably or definitely take palbociclib again (94\%), and nearly all felt that the medication's benefits had met or exceeded their expectations.

\section{Summary points}

- Prior to early 2015, no new treatment options for advanced or metastatic breast cancer patients had been introduced since aromatase inhibitors more than a decade ago.

- Three targeted treatments have been approved in multiple countries for first-line treatment of hormone receptor-positive (HR+)/HER-negative (HER-) advanced breast cancer in combination with aromatase inhibitors or letrozole.

- Little is known about patient experiences and satisfaction with palbociclib combination treatment post approval in real-world settings.

- A validated measure of cancer treatment patient satisfaction, the Cancer Therapy Satisfaction Questionnaire was administered to gauge patient expectations, feelings about side effects and satisfaction with combination therapy in six countries.

- Most patients across all six countries (76.8\%) felt their treatment would help them return to a normal life.

- Regardless of which combination therapy patients were taking or severity of disease course, $92.2 \%$ reported that side effects were as expected or better than expected.

- Overwhelmingly, patients reported they would probably or definitely take palbociclib again (93.5\%), and nearly all felt that the medication's benefits had met or exceeded their expectations.

- This study provides evidence from a real-world setting that patients with advanced breast cancer taking combination therapy are satisfied with their treatment.

- The data gathered in this study could assist physician-patient conversations about patient experiences with the risks, benefits and side effects of biologic treatments for advanced and metastatic breast cancer.

\author{
Acknowledgements \\ Global Perspectives (Norwich, UK) conducted the survey under contract from RTI Health Solutions.
}


the study. D Mitra and S Iyer are employees of Pfizer, Inc. The authors have no other relevant affiliations or financial involvement with any organization or entity with a financial interest in or financial conflict with the subject matter or materials discussed in the manuscript apart from those disclosed.

K Hede of RTI Health Solutions provided medical writing services, which were funded by Pfizer, Inc.

\section{Ethical conduct of research}

RTI International's institutional review board (IRB) reviewed the study design and questionnaire and provided an exemption for the study. The study protocol conformed to the ethical guidelines of the 1975 Declaration of Helsinki. For Canada, central IRB approval was obtained. For Germany, a study notification to the Federal Institute for Drugs and Medical Devices (Bundesinstitut für Arzneimittel und Medizinprodukte, BfArM) was submitted, and approval from Freibeurger Ethik-Kommission International (FEKI) was obtained for Germany, Denmark, and the Netherlands. Separate ethics submissions were not required in Argentina, Denmark or the Netherlands.

\section{Open access}

This work is licensed under the Attribution-NonCommercial-NoDerivatives 4.0 Unported License. To view a copy of this license, visit http://creativecommons.org/licenses/by-nc-nd/4.0/

\section{References}

Papers of special note have been highlighted as: $\bullet$ of interest; $\bullet \bullet$ of considerable interest

1. Ferlay J, Soerjomataram I, Dikshit R et al. Cancer incidence and mortality worldwide: sources, methods and major patterns in GLOBOCAN 2012. Int. J. Cancer 136(5), e359-e386 (2015).

2. Ferlay J, Soerjomataram I, Ervik M et al. GLOBOCAN 2012 v1.0, Cancer Incidence and Mortality Worldwide: IARC CancerBase No. 11 (2013). http://globocan.iarc.fr

3. Johnson RH, Chien FL, Bleyer A. Incidence of breast cancer with distant involvement among women in the United States, 1976 to 2009. JAMA 309(8), 800-805 (2013).

4. Walters S, Maringe C, Butler J et al. Breast cancer survival and stage at diagnosis in Australia, Canada, Denmark, Norway, Sweden and the UK, 2000-2007: a population-based study. Br. J. Cancer 108(5), 1195-1208 (2013).

5. Mariotto AB, Etzioni R, Hurlbert M, Penberthy L, Mayer M. Estimation of the number of women living with metastatic breast cancer in the United States. Cancer Epidemiol. Biomarkers Prev. 26(6), 809-815 (2017).

6. NCI. Cancer stat facts: female breast cancer (2017). https://seer.cancer.gov/statfacts/html/breast.html

7. Howlader N, Altekruse SF, Li CI et al. US incidence of breast cancer subtypes defined by joint hormone receptor and HER2 status. J. Natl Cancer Inst. 106(5), 1-8 (2014).

8. Finn RS, Crown JP, Lang I et al. The cyclin-dependent kinase 4/6 inhibitor palbociclib in combination with letrozole versus letrozole alone as first-line treatment of oestrogen receptor-positive, HER2-negative, advanced breast cancer (PALOMA-1/TRIO-18): a randomised Phase 2 study. Lancet Oncol. 16(1), 25-35 (2015).

9. Finn RS, Martin M, Rugo HS et al. Palbociclib and letrozole in advanced breast cancer. N. Engl. J. Med. 375(20), 1925-1936 (2016).

-• Primary report of Phase III clinical trial results of palbociclib + letrozole, therapies included in this study.

10. Cristofanilli M, Turner NC, Bondarenko I et al. Fulvestrant plus palbociclib versus fulvestrant plus placebo for treatment of hormone-receptor-positive, HER2-negative metastatic breast cancer that progressed on previous endocrine therapy (PALOMA-3): final analysis of the multicentre, double-blind, Phase 3 randomised controlled trial. Lancet Oncol. 17(4), 425-439 (2016).

-. Primary report of Phase III clinical results for palbociclib + fulvestrant therapies included in this study.

11. Kwapisz D. Cyclin-dependent kinase 4/6 inhibitors in breast cancer: palbociclib, ribociclib, and abemaciclib. Breast Cancer Res. Treat. 166(1), 41-54 (2017).

12. US FDA. Accelereted approval of new drug application for Ibrance (palbociclib) (2015). www.accessdata.fda.gov/drugsatfda_docs/appletter/2015/207103orig1s000ltr.pdf

13. US FDA. New drug application approval for KISQALI (ribociclib) (2017). www.accessdata.fda.gov/drugsatfda_docs/appletter/2017/209092s000ltredt.pdf

14. US FDA. New drug application approval for Verzenio (abemaciclib) (2017). www.accessdata.fda.gov/drugsatfda_docs/appletter/2017/208716orig1s000ltr.pdf

15. Vaz-Luis I, O’Neill A, Sepucha K et al. Survival benefit needed to undergo chemotherapy: patient and physician preferences. Cancer 123(15), 2821-2828 (2017).

16. Johnson P, Bancroft T, Barron R et al. Discrete choice experiment to estimate breast cancer patients' preferences and willingness to pay for prophylactic granulocyte colony-stimulating factors. Value Health 17(4), 380-389 (2014). 
17. daCosta DiBonaventura M, Copher R, Basurto E, Faria C, Lorenzo R. Patient preferences and treatment adherence among women diagnosed with metastatic breast cancer. Am. Health Drug Benefits 7(7), 386-396 (2014).

18. Beusterien K, Grinspan J, Tencer T, Brufsky A, Visovsky C. Patient preferences for chemotherapies used in breast cancer. Int. J. Womens Health 4, 279-287 (2012).

19. Muller V, Fuxius S, Steffens CC et al. Quality of life under capecitabine (Xeloda(R)) in patients with metastatic breast cancer: data from a german non-interventional surveillance study. Oncol. Res. Treat. 37(12), 748-755 (2014).

20. de Ligt KM, Spronk PER, van Bommel ACM, Vrancken Peeters M, Siesling S, Smorenburg CH. Patients' experiences with decisions on timing of chemotherapy for breast cancer. Breast 37, 99-106 (2018).

21. De Courcy J, Wood R, Mitra D, Iyer S. Satisfaction with cancer treatments in HR+/HER2- metastatic breast cancer patients in a real world setting. Presented at: ESMO 2016 Congress. Copenhagen, Denmark, 10 October 2016.

- A real-world study of treatment satisfaction in a similar patient population.

22. Abetz L, Coombs JH, Keininger DL et al. Development of the cancer therapy satisfaction questionnaire: item generation and content validity testing. Value Health. 8(Suppl. 1), S41-S53 (2005).

- Presents development and validation of the CTSQ instrument method.

23. Trask PC, Tellefsen C, Espindle D, Getter C, Hsu MA. Psychometric validation of the cancer therapy satisfaction questionnaire. Value Health 11(4), 669-679 (2008).

- Presents further validation of the CTSQ methodology.

24. Pfizer Patient Reported Outcomes. Administration and scoring guide for the Cancer Therapy Satisfaction Questionnaire (CTSQ) (2006). www.pfizerpatientreportedoutcomes.com/system/files/pdf/scoring_ctsq.pdf

25. Pew Research Center. Questionnaire design (2017). www.pewresearch.org/methodology/u-s-survey-research/questionnaire-design/

26. Martinez KA, Kurian AW, Hawley ST, Jagsi R. How can we best respect patient autonomy in breast cancer treatment decisions? Breast Cancer Manag. 4(1), 53-64 (2015).

27. Katz SJ, Belkora J, Elwyn G. Shared decision making for treatment of cancer: challenges and opportunities. J. Oncol. Pract. 10(3), 206-208 (2014).

- Presents context for the importance of shared decision making between patients and caregivers.

28. Chewning B, Bylund C, Shah B, Arora NK, Gueguen JA, Makoul G. Patient preferences for shared decisions: a systematic review. Patient Educ. Couns. 86(1), 9-18 (2012).

- Presents an overview of research on systematic capturing of patient preferences.

29. Zanotti G, Hunger M, Perkins JJ, Horblyuk R, Martin M. Treatment and patient burden in ER+ HER2- metastatic breast cancer: results from a US Physician Survey (2016). www.ispor.org/research_pdfs/52/pdffiles/PCN208.pdf 